\title{
Reflexiones sobre la Biosíntesis de la Morfina y el Parkinson Idiopático
}

\section{Reflections on the biosynthesis of morphine and idiopathic Parkinson's disease}

\author{
José Perea-Sasiaín
}

E- mail: josepesa@gmail.com,

Recibido 11 Abril 2007/Enviado para Modificación 25 Abril 2007/Aceptado 3 Mayo 2007

\section{RESUMEN}

Con base en un caso personal estudiado en forma diaria y continua se deduce que la enfermedad de Parkinson idiopática es una autointoxicación endógena por alteraciones en la biosíntesis de la morfina, y se propone la determinación de los niveles de ésta en la sangre tanto en los parkinsonianos antes de cualquier tratamiento y, con el fin de establecer su diagnóstico preclínico, en los depresivos que constituyen el grupo mas afectado por la enfermedad de Parkinson.

Palabras Clave: Biosíntesis, morfina, Parkinson, diagnóstico (fuente: DeCS, BIREME).

\section{ABSTRACT}

Based on a day-by-day, in-depth study of a clinical case, it was deduced that Parkinson's disease results in autointoxication, due to damage to morphine biosynthesis. Blood morphine levels should be studied in patients suffering from Parkinson's disease before treatment as well as in depressive patients (being the group most affected by Parkinson's disease) to achieve early preclinical diagnosis.

Key Words: Biosynthesis, morphine, Parkinson's disease, diagnosis (source: $\mathrm{MeSH}$, NLM).

$\mathrm{E}$ l pasado año se cumplieron dos siglos de la primera publicación y este año ciento noventa de la última de Sertürner sobre la extracción, el aislamiento y la caracterización de la morfina a partir del opio, látex extraído y desecado de los frutos inmaduros de la adormidera, o en Colombia amapola, Papaver somniferum L (1). Solo otra especie en el reino vegetal produce morfina Papaver setigerum DC que unos consideran una sinonimia y otros que la primera son variedades de cultivo. Se debe destacar la habilidad del hombre 
para detectar sus efectos, domesticarla, usarla y abusar finalmente de sus principios activos.

En 1903 Mavrojannis (2) postuló la síntesis de morfina por las ratas que utilizó para estudios sobre su acción cataléptica; Davis propuso la formación de morfina por los alcohólicos como explicación a su adicción (3). Casi simultáneamente con las publicaciones sobre los péptidos endorfínicos, dos grupos detectaron por medio de radioimmunoensayos una sustancia que inicialmente consideraron similar y posteriormente caracterizaron como morfina endógena $(4,5)$. Se ha encontrado morfina en varias especies de animales y en particular en las ratas de laboratorio y en el hombre (6). Poeaknapo (7) ha demostrado su síntesis endógena mas allá de las dudas sembradas por la propuesta de que la morfina hallada en la leche (8), provenía de las lechugas, o del heno, y las analizó en ambiente separado para evitar la presencia de trazas de morfina en sus laboratorios (7). El grupo que mayor cantidad de publicaciones ha realizado sobre morfina en animales lo lidera Stefano (9).

A la edad de 84 años mi madre inició el temblor digital clásico de la enfermedad de Parkinson idiopática (EPI) que fue su molestia principal durante cuatro años, pues la lentitud y rigidez progresivas eran moderadas y no le impedían moverse. Una crisis emocional agravó el curso clínico y ameritó el comienzo de terapia con levodopa-carbidopa en pequeñas dosis. Esta medicación le permitía movilidad diurna con ayuda y su administración nocturna dormir. Leí un reporte de Matsubara (10) sobre la excreción de grandes cantidades de codeína y la parte proporcional de morfina por las personas a quienes administraron levodopa y ello me bastó para aceptar la existencia de síntesis endógena de morfina en el ser humano y ligarla automáticamente con el sueño profundo y reparador que lograba mi madre en la noche.

Parkinson describió la parálisis agitante (11) y Charcot con reservas sobre esos términos (12) propuso darle el nombre de ese emérito autor. Mi madre presentó muchos otros signos y síntomas de los que afectan a los parkinsonianos y menguan progresiva e inexorablemente su vitalidad: alteración del sueño, lentitud y tropiezos al andar con pasos cortos, depresión ante su enfermedad, seborrea, respiración superficial, babeo, dificultad al deglutir, incrementada por una esofagitis de reflujo, estreñimiento acentuado por la dieta blanda y de poco residuo. La excelente supervisión neurológica y de rehabilitación junto con enfermería y fisioterapia en su propio entorno hicieron menos agobiante su enfermedad hasta la rápida bronconeumonía terminal después de diez años de curso clínico. 
Los parkinsonismos producidos mediante tóxicos (MPTP, 6-hidroxidopamina, paraquat, rotenona, monóxido de carbono) que simulan la EPI por uno o mas de los signos motores no le son equiparables pues "el Parkinsonismo Tóxico bajo ninguna circunstancia se relaciona con la enfermedad de Parkinson o Parkinson Idiopático" (13) y su uso para el diseño de medicamentos que prevengan el progreso de la enfermedad ha llevado a un callejón sin salida (14) ante la falta de efecto para modificar el curso clínico en humanos del TCH346 una sustancia que prevenía la acción del MPTP tanto in vitro como en modelos de animales $(15,16)$.

Los parkinsonismos traumáticos de boxeadores (17) a quienes golpearon abusivamente el rostro y los resultantes de la encefalitis letárgica (18) sin ser casos de EPI, ameritan una prevención más fácil que ésta: la ilegalización del boxeo y la prevención de dicha encefalitis.

Más allá de lo que "se postula como probable etiología" (13) la EPI es casi con plena seguridad una autointoxicación (19). Se admite que la falla neuroquímica que determina los signos motores mas graves de la EPI es la bajísima producción de dopamina en el encéfalo y en particular las neuronas de la sustancia negra (SN) debida a la disminución extrema en la actividad de una ortofenolasa la tirosinhidroxilasa $(\mathrm{TH})$ que forma levodopa a partir de la tirosina (20). Lo anterior explica la excelente respuesta inicial a la levodopa pero estamos convencidos de que el metabolismo secundario de la dopamina, que lleva a la formación de codeína y oripavina precursores directos de la morfina en Papaver somniferum L y en los animales, está tambien alterado y en especial en las etapas que siguen a la formación del anillo isoquinolínico de la norlaudanosolina o tetrahidropapaverolina (THP) con cuatro grupos hidroxilo 2 de los cuales se metilan selectivamente al igual que el nitrógeno del heterociclo para llegar a la S-reticulina. Posteriormente dos metilos se eliminan al pasar de la tebaína a la morfina lo que deja un balance final de las metilaciones en el uso de un metilo. La administración dosificada de metionina agravó en forma perceptible clínicamente a los enfermos a quienes les fue administrada (21) aunque DiRocco (22) describió un efecto antidepresivo con S-adenosilmetionina (SAM) sin reportar deterioro en los signos de 13 pacientes parkinsonianos. La hiperespecialización, y mas particularmente en el área de la neuropsiquiatría, lleva en ocasiones a pasar por alto signos de enfermedad fuera del área propia del especialista; es así como Hoehn y Yahr (18) estimaban en un 4 \% la presencia de depresión en sus casos de EPI y $12 \%$ en los de parkinsonismos postencefalíticos. En la actualidad las cifras varían según los autores entre el 20 \% (23) y el 40 \% (24) de depresión en la EPI. De comprobarse la hipótesis 
de estar la síntesis de morfina endógena alterada en los parkinsonianos, los depresivos constituyen el grupo mas indicado para estimar la producción de morfina en particular a nivel del sistema nervioso central (SNC) en casos de EPI preclínico. El grupo de Kerala en la India no detectó morfina en la sangre de enfermos con EPI cuando sus métodos de análisis permitieron la cuantificación en personas normales o con otras enfermedades del sistema nervioso (25). Otros análisis de morfina en parkinsonianos se han hecho cuando ya están en tratamiento (26) y la levodopa aumenta los niveles de morfina como se puede deducir de los resultados de Matsubara (10). Ese autor considera que la N-metilación, en particular de carbolinas, derivadas del anillo indol proveniente del triptófano, serían agentes causales endógenos $(27,28)$.

Por lo tanto es urgente establecer cuales son los niveles normales de morfina en la sangre humana y compararlos con los de parkinsonianos en etapas iniciales. Para tomar la muestra de sangre se debe establecer primero los factores dietéticos que determinan la gran excreción en relación con la resultante de una dieta líquida de sustitución (29) y hacerlo teniendo en cuenta el horario normal de sueño de la persona pues Horak encontró en sus ratonas albinas de laboratorio variaciones amplias de los niveles de morfina en órganos a la mañana o a la tarde (30). Igualmente, para establecer que parte de la morfina proviene del encéfalo, deben desarrollarse pruebas dando previamente un inhibidor de la dopadecarboxilasa que no atraviese la barrera hematoencefálica (carbidopa, benserazida,etc.) y una segunda prueba dando el anterior y una cantidad bien dosificada de levodopa.

Estudios para correlacionar los niveles de morfina en la sangre con el genoma $(31,32)$ podrían indicar las bases genéticas de la alteración en su síntesis.

Se admite que el deterioro de las neuronas de la SN se presenta y va aumentando muchos años antes de aparecer signos clínicos de la EPI; en este período se deberían instaurar medidas preventivas que permitan un mayor control voluntario de la motricidad, dirigidas a una mejor relajación muscular, caminar con dinámica adecuada segura, un refuerzo de todos los grupos musculares extensores y en particular los de la columna vertebral y de los brazos, la mayor bilateralización y coordinación posible.

Se debería estudiar el efecto de dietas con cantidad adecuada de metionina y de aminoácidos aromáticos pero sin exceso de ellos y medidas antidepresivas de tipo estrictamente psicológico preferiblemente en grupo para mejorar la respuesta anímica frente a la EPI. 
El hallazgo de morfina en líneas tumorales humanas in vitro (33) así como en gliomas (34) y su aumento en el líquido cefalorraquídeo de enfermos con metástasis tumorales meníngeas $(6,35)$ abre un gran área de investigación para establecer que relación tiene la producción de morfina por tumores malignos con la baja respuesta inmunitaria y la falta de dolor en la mayoría de ellos antes de propagarse a sitios muy sensibles. Otra incógnita que se abre es si los tumores que producen morfina excretarían parte de ella a la sangre y en caso tal a que nivel estaría. Los tumores mas llamativos para este tipo de estudio serían los adenocarcinomas mamarios teniendo en cuenta la excreción de morfina en la leche (8).

Finalmente debe estudiarse como afecta la morfina exógena la síntesis endógena. Los morfinómanos alteran a tal punto su propia producción y generan tal cantidad de síntomas y signos molestos que los llevan a depender de dosis masivas externas para aliviarlos y llegan a cualquier extremo para obtenerlas. El enorme costo social del narcotráfico se podría ir reduciendo a medida que los estudios sobre la biosíntesis de la morfina en el hombre dieran resultados aplicables a eliminar en la práctica el síndrome de abstinencia mientras llega el momento en el cual todo ser humano entienda la falsedad y el peligro de los paraísos artificiales como señala magistralmente el cuento fabuloso de Carlo Lorenzini

Agradecimientos. A los profesores Pablo Lorenzana Pombo y Héctor Tejada (q.e.p.d.) por sus visitas a domicilio y sus acertadas recomendaciones y a doña María Teresa Acosta por su buena fisioterapia. Al Dr. Eduardo Arciniegas por leer el manuscrito y sugerir el título de este ensayo.

\section{REFERENCIAS}

1. Huxtable RJ, Schwarz SK. The isolation of morphine - first principles in science and ethics. Mol Interv 2001;1:189-91.

2. Mavrojannis M. L'action cataleptique de la morphine chez les rats. Contribution à la théorie toxique de la catalepsie. C R Soc Biol 1903;55:1092-4.

3. Davis VE, Walker MJ. Alcohol, amines, and alkaloids: a possible biochemical basis for alcohol addiction. Science 1970;167:1005-7.

4. Gintzler AR, Levy A, Spector S. Antibodies as a means of isolating and characterizing biologically active substances: Presence of a non-peptide, morphine-like compound in the central nervous system. Proc Natl Acad Sci USA 1976; 73:2132-6. 
5. Weitz CJ, Lowney LI, Faull KF, Feistner G. Morphine and codeine from mammalian brain. Proc Natl Acad Sci USA 1986; 83:9784-8.

6. Shorr J, Foley K, Spector S. Presence of a non-peptide morphine-like compound in human cerebrospinal fluid. Life Sci 1978;23:2057-62.

7. Poeaknapo C. Mammalian morphine: de novo formation of morphine in human cells. Med Sci Monit 2005; 11:MS6-17.

8. Hazum E, Sabatka JJ, Chang KJ, Findlay JWA, Cuatrecasas P. Morphine in cow and human milk: could dietary morphine constitute a ligand for specific morphine (?) receptors? Science 1981; 213:1010-2.

9. Kream RM, Stefano GB. De novo biosynthesis of morphine in animal cells-based model. Med Sci Monitor 2006; 12(10): 207-19.

10. Matsubara K, Fukushima S, Akane H, Kobayashi S, Shiono H. Increase urinary morphine, codeine and tetrahydropapaveroline in parkinsonian patient undergoing L-3,4-dihydroxyphenylalanine therapy: a possible biosynthetic pathway of morphine from L-3,4-dihydroxyphenylalanine in humans. J Pharmacol Exp Ther 1992; 260:974-8.

11. Parkinson J. An essay on the shaking palsy. Sherwood, Neely, Jones. London, 1817. Reprinted in J Neuropsychiatry Clin Neurosci 2002;14:223-36.

12. Goetz CG. Charcot on Parkinson's disease. Mov Disord 1986;1:27-32.

13. Uribe MG, Rueda MG. Parkinsonismo de etiología tóxica. En: Neurotoxicología, Uribe Granja MG. Asociación Colombiana de Toxicología (Ed.), Bogotá: ELibris; 2000.

14. Lang AE. Neuroprotection in Parkinson's disease: and now for something completely different? Lancet Neurology;2006;5:990-1.

15. Olanow CW, Schapira AHV, LeWitt PA, Kieburtz K, Sauer D, Olivieri G, Pohlmann H, Hubble J. TCH346 as a neuroprotective drug in Parkinson's disease: a double blind, randomised, controlled trial. Lancet Neurol 2006;5:1013-20.

16. Waldmeier P, Bozyczko-Coyne D, Williams M, Vaught JL. Recent clinical failures in Parkinson's disease with apoptosis inhibitors underline the need for a paradigm shift in drug discovery for neurodegenerative diseases. Biochem Pharmacol 2006;72:1197-206.

17. Unterharnscheidt F, A neurologist's reflections on boxing. V. Conclude remarks. Rev Neurol 1995 Sep-Oct;23(123):1027-32.

18. Hoehn M, Yahr MD. Parkinsonism: onset, progression and mortality. Neurology 1967;17:427-42.

19. Williams AC, Ramsden DB. Autotoxicity, methylation and a road to the prevention of Parkinson's disease. J Clin Neurosci 2005 Jan;12(1):6-11.

20. Haavik J, Tosta K. Tyrosine hydroxylase and Parkinson's disease. Molecular Neurobiology 1998;16: 285-309.

21. Pearce LA, Waterbury LD. L-methionine: a possible levodopa antagonist. Neurology 1974;24:840-1. 
22. Di Rocco A, Rogers JD, Brown R, Werner P, Bottiglieri T. S-Adenosyl-Methionine improves depression in patients with Parkinson's disease in an open-label clinical trial. Mov Disord 2000 Nov;15(6):1225-9.

23. Lauterbach EC. The neuropsychiatry of Parkinson's disease and related disorders. Psychiatr Clin North Am 2004;27:801-25.

24. Mayeux R, Stern Y, Williams JB, Cote L, Frantz A, Dyrenfurth I. Clinical and biochemical features of depression in Parkinson's disease. Am J Psychiatry 1986; 143:756-9.

25. Kurup RK, Kurup PA. Hypothalamic digoxin-mediated model for Parkinson's disease. Int J Neurosci 2003 Apr;113(4):515-36.

26. Cadet P, Zhu W, Mantione KJ, Rymer M, Dardik I, Reisman S, Hagberg S, Stefano GB. Cyclic exercise induces anti-inflammatory signal molecule increases in the plasma of Parkinson's patients. Int J Mol Med 2003 Oct;12(4):485-92.

27. Matsubara K, Gonda T, Sawada H, Uezono T, Kobayashi Y, Kawamura T, Ohtaki K, Kimura K, Akaike A. Endogenously occurring beta-carbolines induces parkinsonism in non-primate animals: a possible causative protoxin in idiopathic Parkinson's disease. J Neurochem 1998;70:727-35.

28. Matsubara K, Aoyama K, Suno W, Awaya T. N-methylation underlying Parkinson's disease. Neurotoxicol Teratol 2002 Sep-Oct;24(5):593-8.

29. Hofmann U, Seefried S, Schweizer E, Ebner T, Mikus G, Eichelbaum M. Highly sensitive gas chromatographic-tandem mass spectrometric method for the determination of morphine and codeine in serum and urine in the femtomolar range. J Chromatogr B Biomed Sci Appl 1999 Apr 30;727(1-2):81-8.

30. Horak P, Haberman F, Spector S. Endogenous morphine and codeine in mice-effect of muramyl peptide. Life Sci 1993;52:PL255-63.

31. Forman MS, Lee VM, Trojanowski JQ. Nosology of Parkinson's disease: looking for the way out of a quackmire. Neuron 2005;47:479-82.

32. Fung HC, Scholz S, Matarin M, Simón-Sánchez J, Hernández D, Britton A, Gibbs JR, Langefeld C, Stiegert MI, Schymick J, Okun MS, Mandel RJ, Fernández HH, Foote KD, Rodríguez RL, Peckham E, Wavrant de Vrieze F, Gwinn-Hardy K, Hardy JA, Singleton A. Genome-wide genotyping in Parkinson's disease and neurologically normal controls; first stage analysis and public release of data. Lancet Neurology 2006;5:911-6.

33. Poeaknapo C, Schmidt J, Brandsch M, Dräger B, Zenk MH. Endogenous formation of morphine in human cells. Proc Natl Acad Sci (USA) 2004;191:14091-6.

34. Olsen P, Rasmussen M, Zhu W, Toennesen E, Stefano GB. Human gliomas contain morphine. Med Sci Monit 2005; 11:MS18-21.

35. Cardinale GJ, Donnerer J, Finck AD, Kantrowitz JD, Oka K, Spector S. Morphine and codeine are endogenous components of human cerebrospinal fluid. Life Sci 1987; 40:301-6. 